\title{
Liver dysfunction as the presenting feature of disseminated cryptococcosis ${ }^{i}$
}

\author{
Shailaja Shukla**, Jyoti Garg ${ }^{a}$, Gunjan Mahajan ${ }^{a}$ and Sunita Sharma ${ }^{a}$ \\ a Department of Pathology, Lady Hardinge, Medical College, New Delhi, India \\ *Corresponding author. email: shukla_shailaja@yahoo.com
}

Disseminated cryptococcosis is rare in immunocompetent individuals with central nervous and respiratory systems being the most common initial sites of involvement. Liver dysfunction as a presenting feature of disseminated cryptococcosis has been reported rarely, with the majority of patients being HIV negative and immunocompetent. We report a case of disseminated cryptococcosis in a three-year-old HIV seronegative, immunocompetent male child presenting with obstructive jaundice and lymphadenopathy. Initial diagnosis was made on liver and lymph node biopsy. Lung, skin, central nervous system (CNS) and urinary tract involvement occurred later in the course of disease. Diagnosis was confirmed by serology and culture.

Keywords: cryptococcosis, disseminated, immunocompetent, jaundice, liver

\section{Introduction}

Cryptococcosis is a life-threatening opportunistic fungal infection affecting predominantly immunocompromised hosts. Other than HIV infection, predisposing conditions include immunosuppressive drugs, solid-organ transplantation, haematologic malignancy, chronic organ failure, cirrhosis, chronic lung disease, and rheumatologic disorders. However, cases have also been reported in immunocompetent hosts. ${ }^{1-4}$ CNS and pulmonary involvement are the most common manifestations of disseminated cryptococcosis. ${ }^{3}$ Although disseminated cryptococcosis can involve every organ in the body, including the liver, hepatic involvement as a presenting feature is rare..$^{5-12}$ We report a case of disseminated cryptococcosis in an HIV seronegative, apparently immunocompetent child with hepatic and lymph node involvement as presenting features that mimicked disseminated tuberculosis and malignancy.

\section{Case report}

A three-year-old boy presented to the Paediatrics OPD of Kalawati Saran Children's Hospital, New Delhi with a three-month history of intermittent high grade fever, abdominal distension and increasing jaundice. On clinical examination, the child was febrile and icteric. He had enlarged cervical and axillary lymph nodes along with hepatomegaly. An initial clinical diagnosis of tuberculosis was made as his mother had a past history of tuberculosis and had taken anti-tubercular treatment for six months. Peripheral blood examination revealed anaemia (haemoglobin: $7.4 \mathrm{~g} / \mathrm{dl}$, reference range: $12.5 \pm 1.5 \mathrm{~g} / \mathrm{dl}$ ) and neutrophilic leucocytosis (total leucocyte count: 24,600 cells/ $\mu \mathrm{l}$, reference range: $10,000 \pm 5,000$ cells/ $\mu \mathrm{l}$; neutrophils: $58 \% ; ~ 14,000$ cells/ $\mu \mathrm{l}$, reference range: $1500-8000$ cells/ $\mu \mathrm{l})$. Liver function tests showed hyperbilirubinaemia (total bilirubin: $4.7 \mathrm{mg} / \mathrm{dl}$, reference range: $0.2-1 \mathrm{mg} / \mathrm{dl}$; conjugated bilirubin: $2.8 \mathrm{mg} / \mathrm{dl}$, reference range: $0-0.2 \mathrm{mg} / \mathrm{dl}$ ) with raised liver enzymes (aspartate transaminase: 151 International Unit (IU), reference range: 10-40 IU; alanine transaminase: $63 \mathrm{IU}$, reference range: 10-45 IU; alkaline phosphatase: 2579 IU, reference range: up to 350 IU). Hepatitis serology profile and Leishmania donovani serology were negative. Chest $x$-ray showed bilateral pulmonary infiltrates. Sputum microscopy for acid fast bacilli was negative. Bactec blood culture showed no growth. The patient was HIV seronegative. Fine needle aspiration cytology (FNAC) from cervical lymph nodes was reported as reactive lymphadenitis. Ultrasonogaphy and computerised tomography (CT) of the abdomen showed hepatosplenomegaly with enlarged periportal and mesenteric lymph nodes suggestive of lymphoma or tuberculosis. Bone marrow aspirate smears were reported as diluted with peripheral blood. During this time the child was being treated with broad spectrum antibiotics without any improvement. Since the patient had generalised lymphadenopathy, hepatosplenomegaly and FNAC from cervical lymph node was non-contributory, a liver biopsy and a periportal lymph node biopsy were performed and sent for histopathological examination. Liver biopsy showed maintained lobular architecture with mild Kupffer cell hyperplasia along with both intracellular and canalicular cholestasis. Portal tracts showed widening and large non-caseating granulomas with numerous, foreign body giant cells. Numerous, variably sized $(5-10 \mu \mathrm{m})$ budding yeast forms of Cryptococcus were noted within these giant cells, as well as lying extracellularly from these cells. These fungi displayed narrow based budding (Figure 1).

Lymph node biopsy also showed similar non-caseating granulomatous inflammation with multiple variably sized budding yeast forms of Cryptococcus which were highlighted by periodic acid Schiff and Grocott's methenamine silver stains (Figures $2 \mathrm{~A}$ and $2 \mathrm{~B}$ )

Retrospective examination of FNAC smears from cervical lymph nodes and bone marrow aspirate smears also showed the presence of few budding yeast forms of fungus with the characteristic halo like capsule highlighted by mucicarmine and India ink stain. During his hospital stay the child developed a cough, respiratory distress and papular lesions on his face. Histological examination of the skin biopsy from these lesions also demonstrated Cryptococcus. CT of his lung showed multiple nodules in perilymphatic distribution. Latex agglutination tests on the cerebrospinal fluid (CSF), serum and urine sample were positive for the Cryptococcal antigen. Diagnosis of disseminated cryptococcosis was confirmed by blood and sputum culture. Serotyping identified the organism strain as Cryptococcus neoformans var. neoformans. The patient was commenced on anti-fungal therapy (Amphotericin B $1 \mathrm{mg} / \mathrm{kg} /$ day for 4 weeks, 5 -fluorocytosine $100 \mathrm{mg} / \mathrm{kg} / \mathrm{day}$ for 2 weeks, followed by fluconazole $6 \mathrm{mg} / \mathrm{kg} /$ day for 8 weeks). Clinical response in the 


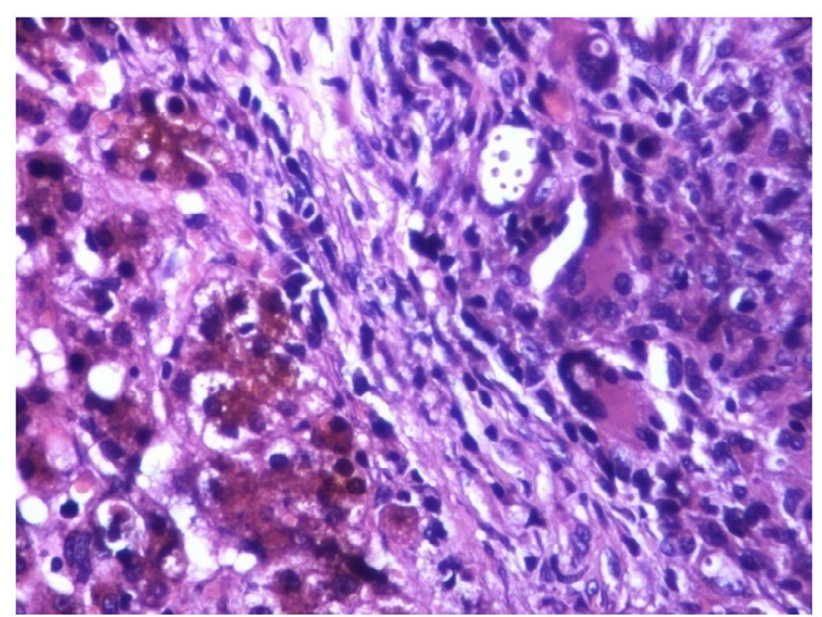

Figure 1: Photomicrograph of liver biopsy showing cholestasis and granulomatous inflammation with yeast forms of Cryptococcus within giant cells as well as lying extracellularly. (H\&E, X 400). also been reported..$^{6,11}$ Liver dysfunction as an initial manifestation of disseminated cryptococcosis has rarely been reported..$^{7,9,12}$ Our patient was HIV negative and apparently immunocompetent. $\mathrm{He}$ had no underlying illness and was not on any immunosuppressive drugs. He had no history of recurrent infections since birth. His CD4 and CD8 lymphocyte counts, immunoglobulin and complement levels were normal. He presented with liver dysfunction and lymphadenopathy, with lung and skin involvement occurring later in the course of the disease.

Hepatic cryptococcosis can manifest as microabscesses, hepatitis, liver failure, cirrhosis or obstructive jaundice. ${ }^{10}$ Our patient presented with obstructive jaundice along with lymphadenopathy. Histopathological findings in liver biopsies from such cases range from granulomatous inflammation to necrosis, hepatitis and cholangitis..$^{10}$ Liver biopsy in our case showed granulomatous inflammation.
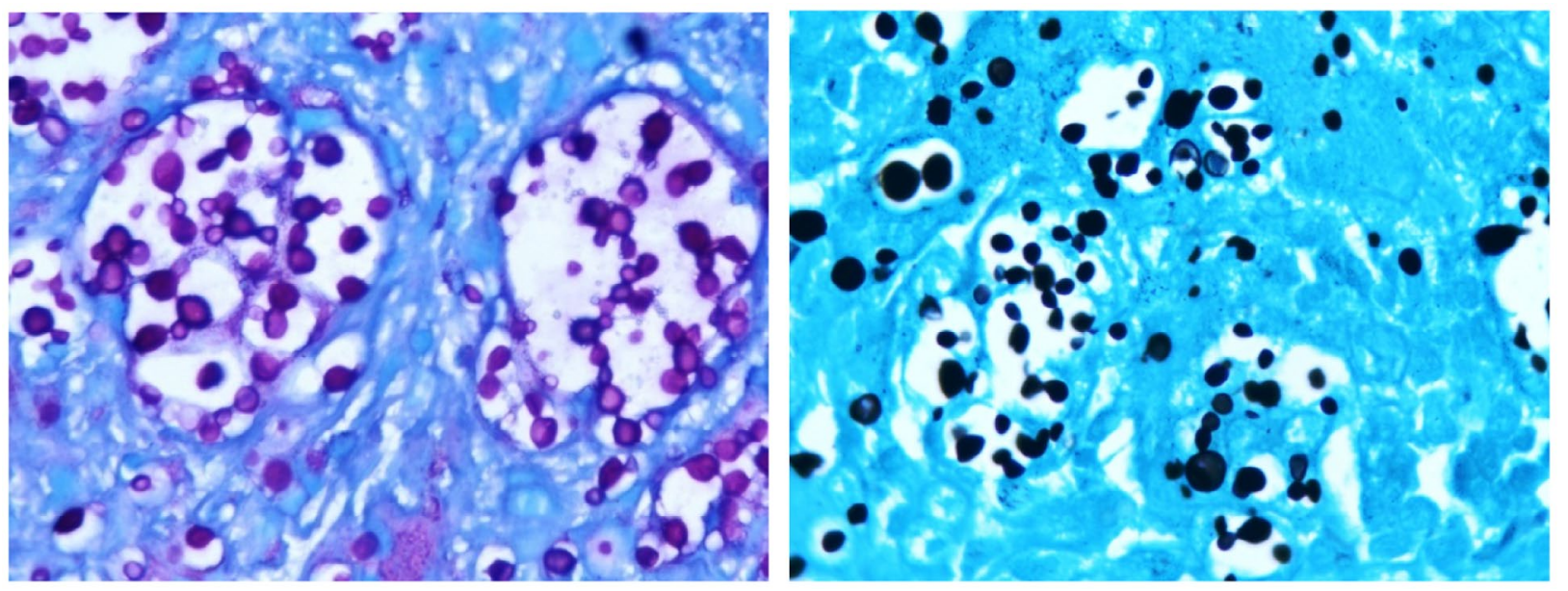

Figure 2: Photomicrograph of lymph node biopsy showing variably sized budding yeast forms of Cryptococcus species highlighted by A) Periodic acid Schiff stain; B) Grocott's methenamine silver stain. (Histochemistry, X1000).

form of a decrease in icterus and resolution of skin lesions was seen within 2 weeks of therapy.

\section{Discussion}

Cryptococcosis is a fungal infection caused by Cryptococcus neoformans. ${ }^{13}$ The fungus exists both in vivo and in vitro in its yeast form. Yeasts are variably sized, ranging from 5 to $25 \mu \mathrm{m}$, demonstrating narrow based budding and a characteristic halo like polysaccharide capsule. Cryptococcus is frequently found in soil that has been contaminated with pigeon droppings; and, the infection spreads through inhalation of aerosolised infectious particles.

Disseminated cryptococcal infections are uncommon and almost always occurs in HIV-infected patients. Clinical presentation of disseminated cryptococcosis is variable and depends on the organ systems involved and immune status of the patient. The most common sites involved are the CNS, lungs and skin. Hepatic involvement is considered rare. Reported rates of hepatic cryptococcosis range from $1-13 \%$ in AIDS patients..$^{10}$ Incidence is rare in HIV seronegative patients with only a few case reports of hepatic cryptococcosis in HIV seronegative patients. ${ }^{5-12}$ In most cases, the liver is involved as a part of the disseminated disease while occasional cases of isolated hepatic cryptococcosis have
The diagnosis of cryptococcosis depends on microscopic demonstration of yeast forms of the organism in CSF, sputum, lymph node aspirate and tissue biopsies. Histochemical stains like India ink, Meyer's mucicarmine, periodic acid Schiff and Grocott's methenamine silver stain are useful for identification of organisms. Definitive diagnosis can be made by culture of organisms using samples such as blood, CSF, sputum, bronchoalveolar lavage and cytological aspirates on fungal media. The latex agglutination test for cryptococcal antigen present in body fluids is also a sensitive and specific test. ${ }^{13}$

Diagnosis of disseminated cryptococcosis is usually delayed in immunocompetent patients due to lack of clinical suspicion. Although hepatic involvement has been reported in the literature, it is not a common presenting manifestation. This case highlights the need to identify liver dysfunction as a possible initial manifestation of fungal infection.

We should include disseminated fungal infections in the differential diagnosis along with tuberculosis and malignancy in patients presenting with fever, hepatomegaly and generalised lymphadenopathy in order to make an early diagnosis and initiate timely treatment. Thorough screening of aspirated material is recommended in order to avoid missing the diagnosis. 
Conflict of Interest - The authors declare that we do not have a commercial or other association that might pose a conflict of interest.

\section{Note}

i. The information contained in this report has not been presented at any meeting to date.

\section{References}

1. Mitha M, Naicker P, Mahida P. Disseminated cryptococcosis in an HIVnegative patient in South Africa: the elusive differential diagnosis. J Infect Dev Ctries. 2010;4:526-529.

2. Suchitha S, Sheeladevi CS, Sunila R, et al. Disseminated cryptococcosis in an immunocompetent patient: a case report. Case Rep Pathol. 2012;2012:652351.

3. Lui $G$, Lee $N$, Ip $M$, et al. Cryptococcosis in apparently immunocompetent patients. QJM. 2006;99:143-151.

4. Jain BB, Bose $D$, Mondal $R$, et al. Disseminated cryptococcosis in an immunocompetent child. Turk Patologi Derg. 2014:01230.

5. Sabesin SM, Fallon HJ, Andriole VT. Hepatic failure as a manifestation of cryptococcosis. Arch Intern Med. 1963;111:661-669.
6. Das BC, Haynes I, Weaver RM. Primary hepatic cryptococcosis. BMJ 1983;287:464.

7. Lin Jl, Kabir MA, Tseng HC, et al. Hepatobiliary dysfunction as the initial manifestation of disseminated cryptococcosis. J Clin Gastroenterol. 1999:28:273-275.

8. Kothari AA, Kothari KA. Hepatobiliary dysfunction as initial manifestation of disseminated cryptococcosis. Indian J Gastroenterol. 2004;23:145-146.

9. Nara S, Sano T, Ojima H. Liver cryptococcosis manifesting as obstructive jaundice in a young immunocompetent man: report of a case. Surg Today. 2008;38:271-274.

10. Liu PY, Yang Y, Shi ZY. Cryptococcal liver abscess: a case report of successful treatment with amphotericin-B and literature review. Jpn J Infect Dis. 2009;62:59-60.

11. Cai X, Liu K, Liang Y, et al. Isolated biliary cryptococcosis manifesting as obstructive jaundice in an immunocompetent adult. Int J Med Sci. 2012;9(3):200-206.

12. Rohtagi A, Aggarwal A, Chabra MK, et al. Disseminated cryptococcosis with hepatic dysfuction as the initial manifestation in an immunocompetent adult. Arch Iran Med. 2013;16(5):303-305.

13. Warkentien T, Crum-Cianflone NF. An update on cryptococcus among HIV-infected patients. Int J STD AIDS. 2010;21:679-684. 